\title{
Magnetoresistance and atomic structure of ultrathin films of $\mathrm{La}_{0.73} \mathrm{Ca}_{0.27} \mathrm{MnO}_{3}$ on $\mathrm{SrTiO}_{3}$
}

\author{
H. W. Zandbergen \\ Material Science Department, Technical University of Delft, Delft, The Netherlands \\ S. Freisem, T. Nojima, ${ }^{*}$ and J. Aarts \\ Kamerlingh Onnes Laboratory, Leiden University, P.O. Box 9504, 2300 RA Leiden, The Netherlands
}

(Received 20 April 1999)

\begin{abstract}
Ultrathin films ( $\approx 6 \mathrm{~nm}$ ) of $\mathrm{La}_{0.73} \mathrm{Ca}_{0.27} \mathrm{MnO}_{3}$ on $\mathrm{SrTiO}_{3}$ exhibit properties quite different from the bulk. They become ferromagnetically ordered around $150 \mathrm{~K}$ but remain insulating, instead of showing the insulatorto-ferromagnetic metal transition which occurs in bulk material or thicker films at this $\mathrm{Ca}$ concentration. Electron microscopy shows that such films are perfectly crystalline but have a crystal structure, imposed by the lattice mismatch with the substrate, which is different from that of the bulk or thick films. An important feature is a Jahn-Teller-like distortion of the $\mathrm{MnO}_{6}$ octahedra which is not present in the bulk and appears to be the cause of the changed physical properties. [S0163-1829(99)09837-9]
\end{abstract}

\section{INTRODUCTION}

Lately, perovskite manganite compounds of the type $A_{1-x} A^{\prime}{ }_{x} \mathrm{MnO}_{3}$ (with $A$ a trivalent rare earth such as $\mathrm{La}$, and $A^{\prime}$ a divalent dopant such as $\mathrm{Sr}$ or $\mathrm{Ca}$ ) have been studied intensively because of the "colossal" magnetoresistance (CMR) effects which are found at temperatures around the combined paramagnetic-ferromagnetic and insulator-metal (IM) transitions. The classical description of this phenomenon involves the so-called double exchange mechanism, ${ }^{1}$ in which hopping of an outer shell electron from a $\mathrm{Mn}^{3+}$ to a $\mathrm{Mn}^{4+}$ site is favored by a parallel alignment of the core spins. Ferromagnetism therefore promotes hopping (high conductivity) and vice versa. However, this mechanism does not correctly account for the magnitude of the observed resistance drops. Another necessary ingredient is electronlattice coupling. ${ }^{2,3}$ For the $\mathrm{Mn}^{3+}$, with three electrons in the energetically lower spin triplet state and the mobile electron in the energetically higher doublet, a Jahn-Teller distortion of the oxygen octahedron can lead to splitting of the doublet; for the $\mathrm{Mn}^{4+}$, the energy of the (empty) doublet can be lowered by coupling to a breathing mode of the lattice. Both effects tend to trap the electron in a polaronlike state, which is in competition with band formation due to ferromagnetic correlations. When lowering the temperature through the ferromagnetic transition at $T_{c}$, the high resistance polaron state breaks up and changes into a lower resistive "ferromagnetic metal" (FM) state. A second important connection between crystal structure and insulator-metal transition lies in the dependence of the Mn-Mn electron transfer on the Mn-O-Mn bond angles, or equivalently on the orientation of the oxygen octahedra with respect to the main crystal axes. This results in a strong dependence of $T_{c}$ on either external pressure or mean $A$-site ionic radius, for which a universal phase diagram (at constant doping) can be constructed. ${ }^{4}$

For films, the situation is more complicated. Maximum MR values in films are usually larger and at lower temperatures ${ }^{5,6}$ than in the equivalent bulk materials (although suitable preparation conditions can lead to "bulklike" behavior ${ }^{7}$ ), which is generally believed to be due to structural disorder from, e.g., nonepitaxial growth or partial strain relaxation. Quite often, the investigated film thicknesses are of the order of $100 \mathrm{~nm}$, making strain relaxation likely. Very thin films $(\approx 10 \mathrm{~nm})$, however, can be uniformly strained by an underlying substrate, and it should be possible to predict their physical properties; these will be different from the changes induced by hydrostatic or chemical pressure, since in-plane (epitaxial) strain leads to an outof-plane strain of different sign. Strain can be used to induce properties outside the bulk phase diagram, as was recently demonstrated for a high- $T_{c}$ perovskite; ${ }^{8}$ for CMR materials, it was predicted that $T_{c}$ is sensitive to biaxial strain. ${ }^{9}$ Here, we extend earlier work on sputtered films of $\mathrm{La}_{0.73} \mathrm{Ca}_{0.27} \mathrm{MnO}_{3}$ ("CMR" regime) ${ }^{6}$ to focus on strain effects from a $\mathrm{SrTiO}_{3}$ (STO) substrate (1\% larger lattice parameter) in the range of very small thickness $(6-12 \mathrm{~nm})$, where relaxation is still minimal. In the earlier work we reported that films with larger thickness $(30-200 \mathrm{~nm})$ show the usual IM transition, but that below $30 \mathrm{~nm}$ the films become increasingly insulating. Now we show from high-resolution electron microscopy (HREM) that the larger substrate lattice parameter induces a crystal structure which is different from the bulk, leading to a ferromagnetic insulating (FI) ground state with still a large MR ratio in high magnetic fields. The $T_{c}$ of the changed structure is lower than that of the bulk but, surprisingly, roughly equal to the $T_{c}$ of the thicker films which show the bulk structure and a metallic ground state. The results emphasize that the effects of even moderate strain can be severe, and that partial strain relaxation is important for thin-film properties.

\section{EXPERIMENTAL}

All films were sputter deposited from ceramic targets of $\mathrm{La}_{1-x} \mathrm{Ca}_{x} \mathrm{MnO}_{3}$ with a nominal composition of $x=0.33$ on STO substrates in a pure oxygen atmosphere of $300 \mathrm{~Pa}$, at a growth temperature of $840{ }^{\circ} \mathrm{C}$ using a (low) growth rate of the order of $0.9 \mathrm{~nm} / \mathrm{min}$. After deposition, each sample was cooled to room temperature without further annealing. This procedure always resulted in films of high crystalline quality 

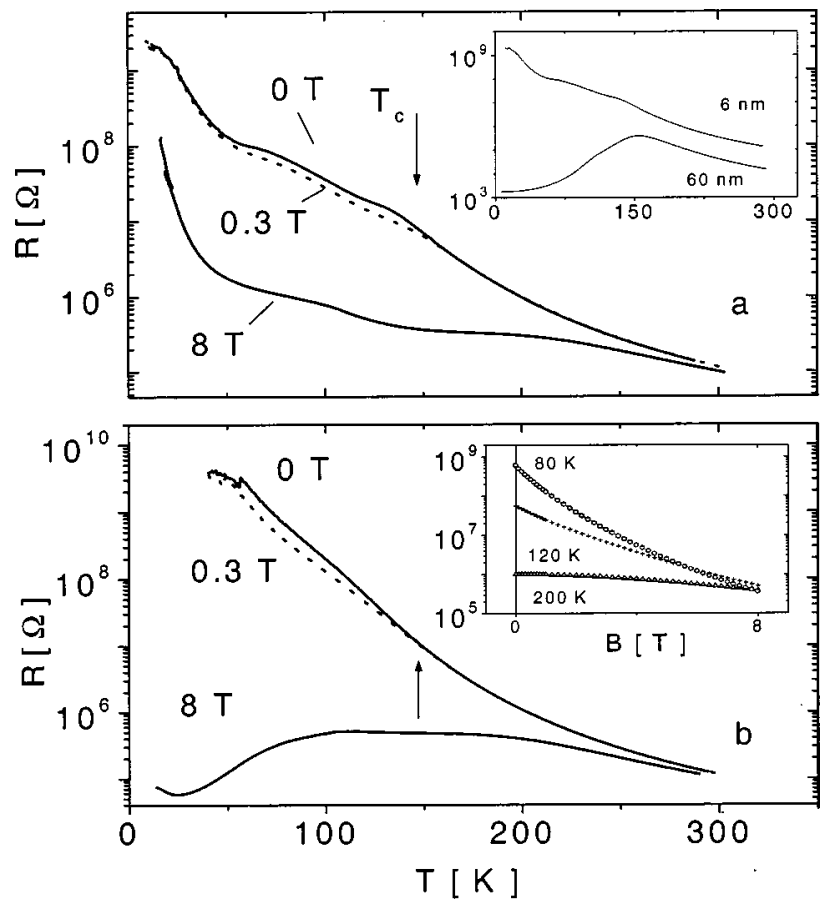

FIG. 1. Resistance on a logarithmic scale versus temperature for films of $\mathrm{La}_{0.73} \mathrm{Ca}_{0.27} \mathrm{MnO}_{3}$ with (a) $d_{s}=6 \mathrm{~nm}$ and (b) $d_{s}=8 \mathrm{~nm}$, in zero field and fields of 0.3 and $8 \mathrm{~T}$ as indicated. The arrows denote the ferromagnetic transition temperatures determined from magnetization. The inset in (a) shows the behavior of the 6-nm film compared to a $60-\mathrm{nm}$ film. The inset in (b) shows the MR behavior for the 8-nm film.

and in very sharp film-substrate interfaces, according to HREM. Given the high oxygen sputter pressure, the isotropic oxygen diffusion, and the small film thickness, it is probable that an equilibrium oxygen content is obtained. The chemical composition of the films was determined by microprobe analysis, which showed a $(\mathrm{La}, \mathrm{Ca}) / \mathrm{Mn}$ ratio of $1: 1$ and a $\mathrm{Ca}$ content of $x=0.27 \% \pm 0.02$. The resistance $R$ was obtained from unpatterned samples with sputtered gold contacts, since patterning was found to lead to degradation of the samples. The area between the voltage contacts was roughly 5 $\times 5 \mathrm{~mm}^{2}$, so that an estimate for the resistivity $\rho$ can be obtained from $\rho=R / d_{s}$, with $d_{s}$ the film thickness. The magnetization $M$ was measured with a commercial superconducting quantum interference device (SQUID) magnetometer. HREM was performed with a Philips CM30UT electron microscope with a field emission gun operated at 300 $\mathrm{kV}$.

\section{RESULTS}

Figure 1(a) shows $R$ versus temperature $T$ of a 6-nm-thick film in zero field and in fields of 0.3 and $8 \mathrm{~T}$. In zero field, $R_{0 T}$ increases by about four orders of magnitude going from 300-4 K. In $0.3 \mathrm{~T}$, a small deviation from the zero-field curve occurs below $150 \mathrm{~K}$, which is the value of $T_{c}$ according to the magnetization. In $8 \mathrm{~T}, R_{8 T}$ increases only slowly with decreasing temperature. Fig. 1(b) shows $R(T)$ of an 8 -nm-thick film for the same fields. The increase in $R_{0 T}$ here is even larger, while $R_{8 T}$ is roughly constant; both films clearly show very high MR ratios. The magnetoresistance is

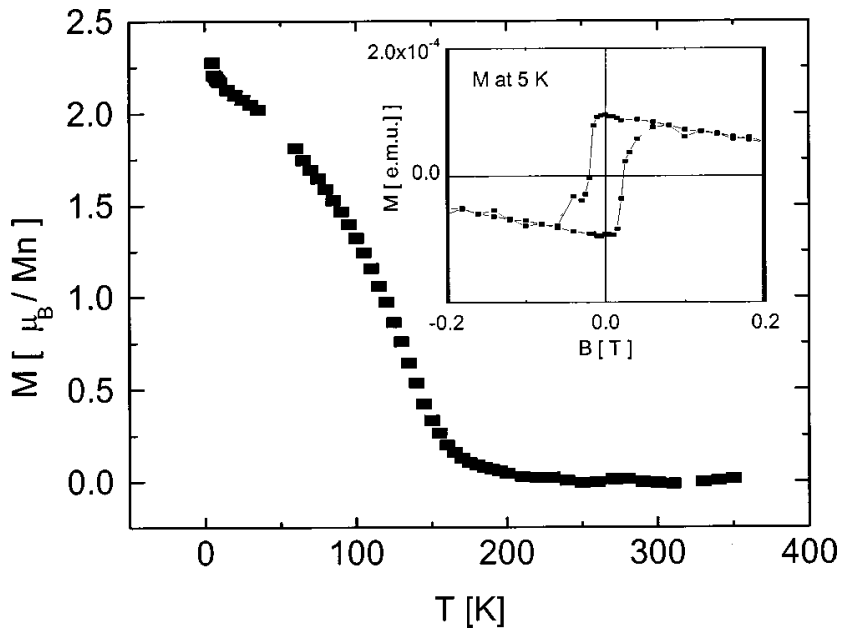

FIG. 2. Magnetization (corrected for substrate contribution) in a field of $0.3 \mathrm{~T}$ versus temperature of the $6-\mathrm{nm}$ film. Inset: magnetization (as measured) versus applied field at $5 \mathrm{~K}$.

flat above $T_{c}$, and monotonous and cusplike below $T_{c}$, as shown in the inset in Fig. 1(b) for 200, 120, and $80 \mathrm{~K}$. The inset in Fig. 1(a) shows the behavior of a typical $60-\mathrm{nm}$ film with the usual IM transition for comparison. In order to ascertain that these films are continuous, they were scanned by electron microscopy over several hundreds of microns; not once was a significant change in film thickness observed. A number of films was investigated in a thickness range of 6-12 nm; $R(T, H)$ did not show hysteresis in warming versus cooling, and only small variations were found. One 6-nm sample showed a small peak in zero field around $70 \mathrm{~K}$, but the lowest resistance value below the peak was still significantly higher than the resistance at $300 \mathrm{~K}$. Figure 2 shows $M$ versus $T$ of the 6-nm sample of Fig. 1(a) measured in $0.3 \mathrm{~T}$, after correction for the magnetization of the substrate. $M$ starts to rise around $150 \mathrm{~K}$, which is taken as $T_{c}$ and reaches a value of about $2.5 \mu_{B} / \mathrm{Mn}$ atom at $5 \mathrm{~K}$. A value lower than expected for the $\mathrm{Mn}^{3+}-\mathrm{Mn}^{4+}$ spin mixture $\left(3.7 \mu_{B} / \mathrm{Mn}\right.$ atom) is often found in films, ${ }^{6}$ and $M$ still increases slowly in higher fields. The inset of Fig. 2 shows the hysteretic magnetization at $5 \mathrm{~K}$, demonstrating that the film is ferromagnetic.

HREM was applied to determine the atomic structure of these very thin films. A distinguishing feature of the bulk structure is that the $\mathrm{MnO}_{6}$ octahedra are tilted around the $b$ axis $\left(8.5^{\circ}\right)$ and the $a$ axis $\left(11^{\circ}\right)$, resulting in a orthorhombic unit cell of dimensions $a_{p} \sqrt{2}, 2 a_{p}, a_{p} \sqrt{2}$, with $a_{p}$ the lattice parameter of the simple cubic unit cell of the ideal perovskite. Figure 3 presents a HREM image of a 6-nm-thick film viewed parallel to the interface along $[100]_{\mathrm{STO}}$ of $\mathrm{SrTiO}_{3}$ (parallel to the [101] direction of $\mathrm{La}_{0.73} \mathrm{Ca}_{0.27} \mathrm{MnO}_{3}$ ). The image shows a $2 a_{p}$ periodicity ( $2 a_{p}$ fringes) along the interface normal, which is related to the $b$ axis of the bulk structure. These fringes are symmetry forbidden, even for dynamic diffraction, but were imaged by a slight symmetrybreaking tilt of the crystal. ${ }^{10}$ At positions indicated by the arrows, defects occur with a shift of the $2 a_{p}$ fringes over $b / 2$. Such defects are observed throughout the film, when viewed along $[100]_{\mathrm{STO}}$. They can be explained either as antiphase boundaries (APB's), which implies that the unit cell shifts over a distance $b / 2$ when going through the bound- 


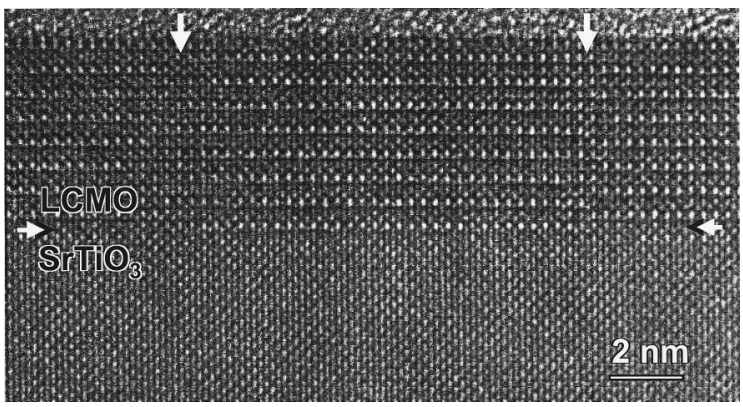

FIG. 3. HREM image of a 6-nm film of $\mathrm{La}_{0.73} \mathrm{Ca}_{0.27} \mathrm{MnO}_{3}$ viewed along $[100]_{\mathrm{STO}}$. In this film as well as in a 12-nm film the $b$ axis is along the interface normal everywhere. The approximate position of the film-substrate interface is indicated by horizontal arrows. Defects are indicated by vertical arrows.

ary, or as twin boundaries (TB's), which implies an interchange of the $a$ and $c$ axis. The issue is resolved using images along $[101]_{\mathrm{STO}}$, which can be along either the $a$ or the $c$ axis of the film. One such image is given in Fig. 4(a). Figure 4(b) (upper part) shows the expected intensity patterns for images viewed along either axis. Figure 4(a) belongs to the $a$ axis oriented pattern, with alternating rows of high and low intensity of the superstructure along the diagonal of the rectangular unit cell. The half period shift in intensity alternations caused by an APB should be found along the same diagonal. Such changes are better visualized by treating the image as shown in Fig. 4(c). Here, every second vertical slice of white dots was displaced perpendicular to the interface over $b / 2$ as indicated in the inset. The resulting picture shows a superstructure similar to the one in the $[100]_{\text {STO }}$ viewing direction and APB's should again be identifiable by shifts of the $2 a_{p}$ fringes. Such shifts are clearly not present here, and actually never observed, although care was taken to image areas considerably larger than the average defect distance. The defects visible in Fig. 3 are therefore due to twinning. Another conclusion can be

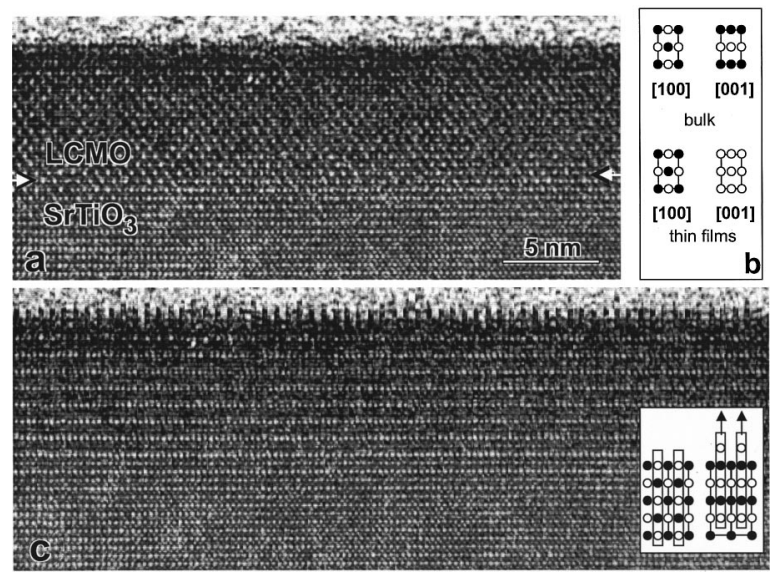

FIG. 4. (a) HREM image of a 6-nm $\mathrm{La}_{0.73} \mathrm{Ca}_{0.27} \mathrm{MnO}_{3}$ film viewed along $[101]_{\mathrm{STO}}$. The approximate position of the filmsubstrate interface is indicated by arrows; (b) intensity patterns for the [100] and [001] directions of the bulk crystal structure (upper panel), and of the observed patterns in the ultrathin films (lower panel); (c) image treated by shifting atomic columns as indicated in the inset.
TABLE I. Mn-O distances (in $\AA$ ) and $\mathrm{Mn}-\mathrm{O}(1)-\mathrm{Mn}$ bond angles (in deg) for $\mathrm{La}_{0.67} \mathrm{Ca}_{0.33} \mathrm{MnO}_{3}$ ( $\mathrm{LCMO}$ ), the films, $\mathrm{La}_{0.88} \mathrm{Sr}_{0.12} \mathrm{MnO}_{3}$ (LSMO), and $\mathrm{LaMnO}_{3}$ (LMO). O(1) is the apical oxygen, $\mathrm{O}(2)$ are the oxygens in the $a-c$ plane.

\begin{tabular}{lcccc}
\hline \hline & LCMO & film & LSMO & LMO \\
\hline $\mathrm{Mn}-\mathrm{O}(1)$ & 1.956 & 1.93 & 1.98 & 1.97 \\
$\mathrm{Mn}-\mathrm{O}(2)$ & 1.960 & 2.09 & 2.04 & 2.18 \\
$\mathrm{Mn}-\mathrm{O}(2)$ & 1.956 & 1.85 & 1.94 & 1.91 \\
$\mathrm{Mn}-\mathrm{O}(1)-\mathrm{Mn}$ & 159.6 & 158.8 & - & - \\
Ref. & 13 & - & 11 & 12 \\
\hline \hline
\end{tabular}

drawn from Fig. 4. In thick films both [100] ( $a$ axis) and [001] ( $c$ axis) patterns are observed, but in the 6-12-nm films only the [100] patterns occur. Moreover, the intensity of the [100] pattern varies from area to area and is in some areas very weak or even absent, correlating very well with the twinning observed in Fig. 3(a). Apparently, [001] patterns lack superstructure intensity variations, as indicated in Fig. 4(b) (lower part), and the conclusion is that these very thin films do not possess a superstructure in the [001] projection. This was verified by performing through-focus checks in HREM mode on many areas, as well as by checking relatively thick areas $(20-200 \mathrm{~nm})$ in electron diffraction mode.

The absence of such a superstructure indicates that - in contrast with bulk $\mathrm{La}_{0.73} \mathrm{Ca}_{0.27} \mathrm{MnO}_{3}$-no significant tilt of the $\mathrm{MnO}_{6}$ octahedra about the $b$ axis is present. However, the presence of a superstructure in the [101] projection cannot be explained by a tilt about the $a$ axis alone. The only other structural change able to produce such superstructure reflections is obtained by shearing oxygen rows along the $c$ axis, alternatingly in positive and negative direction, which yields an elongation of the square of oxygens in the $a-c$ plane. This is a Jahn-Teller-like distortion, but now induced by the substrate. Structure refinements using electron diffraction data (see Refs. 11-13) yield Mn-O distances as given in Table I, together with numbers for the bulk compounds $\mathrm{La}_{0.7} \mathrm{Ca}_{0.3} \mathrm{MnO}_{3}$ (FM ground state), $\mathrm{La}_{0.88} \mathrm{Sr}_{0.12} \mathrm{MnO}_{3}$ (FI), and $\mathrm{LaMnO}_{3}$ (AFI). Clearly, the distortions in the film are similar in value to those in the bulk compounds with an insulating ground state.

\section{DISCUSSION}

The reason for the change of structure lies in the larger lattice of STO $\left(a_{p}=0.391 \mathrm{~nm}\right)$ compared to bulk $\mathrm{La}_{0.73} \mathrm{Ca}_{0.27} \mathrm{MnO}_{3}\left(a_{p}=0.387 \mathrm{~nm}\right)$, which necessitates inplane expansion of the film. The associated volume increase is partly compensated by a lattice contraction along the interface normal ( $a_{p}=0.384$ along $b$ ), but also by the loss of the tilt about $b$, which in the bulk leads to a smaller unit cell for a better accomodation of the $\mathrm{La}(\mathrm{Ca})$ ion. However, the in-plane expansion does not require deformation of the oxygen squares; that it happens shows how close the bulk structure is to a Jahn-Teller-like lattice instability. This is the more striking since the structure does not appear very favorable regarding elastic energies. Since the Mn-Mn distance along the $c$ axis now differs from the distance along $a$, a misfit energy occurs. This energy is reduced by a frequent 
interchange of both axes, through the generation of the observed (101) mirror plane boundaries. A further consequence of the structure is that a contraction of the lattice for growth on a smaller substrate (e.g., $\mathrm{LaAlO}_{3}$ ) may not be achieved as easily, since it needs a larger tilt about $b$, which is inhibited by the $\mathrm{La}(\mathrm{Ca})$ ion. As a matter of fact, growth on $\mathrm{LaAlO}_{3}$, with strong island formation, is found to be qualitatively different from the layer-by-layer growth on STO, ${ }^{14}$ indicating such steric hindrance may be at play. A final remark on the structure concerns relaxation effects. The average spacing of TB's in a $12-\mathrm{nm}$ film (30 $\mathrm{nm}$ with a large variation) is considerably larger than that in the 6-nm film (about $10 \mathrm{~nm}$ with a small variation). In films of $40 \mathrm{~nm}$ and thicker TB's are rare or absent; instead, misfit dislocations occur frequently, indicating that the misfit strain is (partly) released, so that the bulk structure can be more closely approached. Images along $[101]_{\text {STO }}$ show domains with typical bulk structure features. The thickness for the onset of these relaxation effects under our growth conditions is therefore about $20 \mathrm{~nm}$.

With respect to the physical properties, to be noted in particular is the value of $T_{c}$, which lies in the range 150$170 \mathrm{~K}$. This is clearly lower than the bulk value of $250 \mathrm{~K}$, but higher than the temperature where FI occurs in the bulk phase diagram for $A_{0.7} A^{\prime}{ }_{0.3} \mathrm{MnO}_{3}$. There, the FI state is reached by fully substituting $\operatorname{Pr}$ for $\mathrm{La}$, which yields a decrease in $\mathrm{Mn}-\mathrm{O}(1)-\mathrm{Mn}$ bond angle $[\mathrm{O}(1)$ is the apical oxy-

*Permanent address: Center for Low Temperature Science, Tohoku University, 2-1-1 Katahira, Aoba-ku, Sendai 980-8577, Japan.

${ }^{1}$ C. Zener, Phys. Rev. 82, 403 (1951); P. W. Anderson and H. Hasegawa, ibid. 100, 675 (1955).

${ }^{2}$ A. J. Millis, P. B. Littlewood, and B. I. Shraiman, Phys. Rev. Lett. 74, 5144 (1995).

${ }^{3}$ A. J. Millis, B. I. Shraiman, and R. Mueller, Phys. Rev. Lett. 77, 175 (1996)

${ }^{4}$ H. Y. Hwang, T. T. M. Palstra, S.-W. Cheong, and B. Batlogg, Phys. Rev. B 52, 15046 (1995); see also A. P. Ramirez, J. Phys.: Condens. Matter 9, 8171 (1997).

${ }^{5}$ S. Jin, T. H. Tiefel, M. McCormack, H. M. O’Bryan, L. H. Chen, R. Ramesh, and D. Schurig, Appl. Phys. Lett. 67, 557 (1995).

${ }^{6}$ J. Aarts, S. Freisem, R. Hendrikx, and H. W. Zandbergen, Appl. Phys. Lett. 72, 2975 (1998).

${ }^{7}$ M. F. Hundley, M. Hawley, R. H. Heffner, Q. X. Jia, J. J. Neumeier, J. Tesmer, J. D. Thompson, and X. D. Wu, Appl. Phys. gen] from $159.5^{\circ}-156.5^{\circ},{ }^{13,14}$ leading to a decrease of the itinerant electron bandwidth which is apparently enough to inhibit the formation of the FM state. In the ultrathin films, the mechanism inducing the FI state is radically different. The Mn-O(1)-Mn bond angle is only slightly lower than in the bulk (see Table I). This probably causes the lower $T_{c}$ since the ferromagnetism is especially sensitive to the strength of the out-of-plane interactions. Note that the change in structure precludes a comparison to theories for strain dependence of $T_{c} .{ }^{9}$ The insulating state is clearly due to the in-plane distortions, which trap the mobile electrons, and can be seen as a strongly increased electron-lattice coupling. Finally, the large MR ratio is very similar to what is found in bulk $\mathrm{Pr}_{0.7} \mathrm{Ca}_{0.3} \mathrm{MnO}_{3},{ }^{4}$ which is also in the FI state; both effects are much larger than the MR of (FI) $\mathrm{La}_{0.88} \mathrm{Sr}_{0.12} \mathrm{MnO}_{3},{ }^{11}$ indicating that the large MR is due to the high doping concentration.

\section{ACKNOWLEDGMENTS}

The authors thank L. Feiner and A. J. Millis for discussions. This work was supported by the Nederlandse Stichting voor Fundamenteel Onderzoek der Materie (FOM). T.N. wishes to acknowledge support from the Japan Society for the Promotion of Science.

Lett. 67, 860 (1995).

${ }^{8}$ J.-P. Locquet, J. Perret, J. Fompeyrine, E. Mächler, J. W. Seo, and G. Van Tendeloo, Nature (London) 394, 453 (1998).

${ }^{9}$ A. J. Millis, T. Darling, and A. Migliori, J. Appl. Phys. 83, 1588 (1998).

${ }^{10}$ H. W. Zandbergen, J. Jansen, S. Freisem, T. Nojima and J. Aarts, Philos. Mag. Lett. (to be published).

${ }^{11}$ D. N. Argyriou, J. F. Mitchell, C. D. Potter, D. G. Hinks, J. D. Jorgensen, and S. D. Bader, Phys. Rev. Lett. 76, 3826 (1996).

${ }^{12}$ F. Moussa, M. Hennion, J. Rodriguez-Carvajal, H. Moudden, L. Pinsard, and A. Revcolevschi, Phys. Rev. B 54, 15149 (1996).

${ }^{13}$ Q. Huang, A. Santoro, J. W. Lynn, R. W. Erwin, J. A. Borchers, J. L. Peng, K. Ghosh, and R. L. Greene, Phys. Rev. B 58, 2684 (1998).

${ }^{14}$ S. Freisem, T. Nojima, R. W. Hendrikx, H. W. Zandbergen, and J. Aarts, Proc. SPIE 3481, 342 (1998). 